\title{
Fatty acid synthase-positive hepatocytes and subsequent steatosis in rat livers by irinotecan
}

\author{
TAKEYUKI SAWANO ${ }^{1}$, TAKESHI SHIMIZU ${ }^{2}$, TOSHIYUKI YAMADA ${ }^{2}$, NAOKI NANASHIMA ${ }^{3}$, \\ TAKUYA MIURA $^{1}$, SATOKO MOROHASHI $^{4}$, DAISUKE KUDO ${ }^{1}$, FENG MAO HUI ${ }^{1,5}$, \\ HIROSHI KIJIMA $^{4}$, KENICHI HAKAMADA ${ }^{1}$ and SHIGEKI TSUCHIDA ${ }^{2}$
}

\begin{abstract}
Departments of ${ }^{1}$ Gastroenterological Surgery and ${ }^{2}$ Biochemistry and Genome Biology, Hirosaki University Graduate School of Medicine; ${ }^{3}$ Department of Medical Technology, Hirosaki University Graduate School of Health Sciences;

${ }^{4}$ Department of Pathology and Bioscience, Hirosaki University Graduate School of Medicine, Hirosaki, Japan
\end{abstract}

Received January 5, 2015; Accepted February 3, 2015

DOI: $10.3892 /$ or.2015.3814

\begin{abstract}
Using a rat model, we investigated factors contributing to the pathogenesis of irinotecan-associated fatty liver disease. Male Sprague-Dawley rats were administered $200 \mathrm{mg} / \mathrm{kg}$ irinotecan by intraperitoneal injection on days 1-4, but not on days 5-7. This schedule was repeated 3 times. Rats were sacrificed 4, 18 and 25 days after the last injection, and liver steatosis was evaluated by hematoxylin and eosin (H\&E) staining, microarray analysis and immunohistochemistry. Panacinar intrahepatocyte vacuoles were absent on days 4 and 25 , but present on day 18 , and this alteration was more prominent around the bile ducts than the central veins. Microarray analysis showed that the expression of genes involved in the synthesis of cholesterol and fatty acids was upregulated on day 4. Immunohistochemistry detected fatty acid synthase (Fasn)strongly positive hepatocytes as well as the activation of liver progenitor cells on day 4 , whereas intracellular vacuoles were
\end{abstract}

Correspondence to: Dr Shigeki Tsuchida, Department of Biochemistry and Genome Biology, Hirosaki University Graduate School of Medicine, Hirosaki 036-8562, Japan

E-mail: tsuchida@cc.hirosaki-u.ac.jp

Present address: ${ }^{5}$ Department of Oncology Surgery, Zhongnan Hospital, Wuhan University, Wuhan, Hubei 430071, P.R. China

Abbreviations: H\&E, hematoxylin and eosin; RT-PCR, real-time polymerase chain reaction; Fasn, fatty acid synthase; CA3, carbonic anhydrase 3; CRLM, colorectal cancer liver metastasis; CPT-11, irinotecan; NASH, nonalcoholic steatohepatitis; Cxcl9, chemokine (C-X-C motif) ligand 9; NAFLD, nonalcoholic fatty liver disease; $\mathrm{CV}$, central veins; BD, bile ducts; Srebp2, sterol regulatory elementbinding protein 2; Insig1, insulin induced gene 1; Scap, SREBP cleavage-activating protein; Eif2ak4, eukaryotic translation initiation factor $2 \alpha$ kinase 4; PAS, periodic acid-Schiff; Egr1, early growth response 1; Nrli3, nuclear receptor subfamily 1, group I, member 3; $\mathrm{CASH}$, chemotherapy-associated steatohepatitis

Key words: fatty acid synthase, liver progenitor cell, Kupffer cell, chemotherapy-associated steatohepatitis, irinotecan evident in carbonic anhydrase 3 (CA3)-positive hepatocytes on day 18. Thus, irinotecan-induced liver steatosis was preceded by Fasn-strongly-positive hepatocytes and liver progenitor cell activation. The magnitude of the decrease in the number of Fasn-strongly positive hepatocytes between days 4 and 18 was similar to that of the increase in the number of CA3-positive hepatocytes accompanying vacuoles.

\section{Introduction}

Colorectal cancer is one of the most commonly reported malignancies, and approximately one-third of patients with advanced colorectal cancer have liver metastasis (1). Although surgical resection is the standard treatment of colorectal cancer liver metastasis (CRLM) (2), <20\% of cases are resectable at the initial diagnosis (3). Therefore, chemotherapy is frequently employed to downsize CRLM prior to surgery. New regimens, including the combination of fluorouracil and calcium folinate with irinotecan (CPT-11) (4) or oxaliplatin (5) have significantly increased tumor response rates to nearly $50 \%$ (6). These regimens followed by liver resection have been shown to improve 5-year survival rates to $\sim 40 \%$, which is similar to that of patients that undergo primary liver resection (7).

However, irinotecan has been associated with the development of chemotherapy-associated steatohepatitis (8) and increases mortality in subsequent hepatic surgery (9). The antitumor activity of irinotecan is dependent on the inhibition of DNA topoisomerase I activity and is activated to SN-38, its active metabolite by carboxylesterase (10). Adverse effects associated with irinotecan such as severe neutropenia have been attributed to the inadequate inactivation of SN-38 (11). The mechanism responsible for the induction of steatohepatitis following the administration of irinotecan has not yet been elucidated. Previous clinical studies suggested that the dose or duration of the drug was not responsible (8), whereas the interval between chemotherapy and subsequent hepatic resection was correlated to the rate at which steatohepatitis was induced (12). These findings suggested that the induction of steatohepatitis may not be due to the direct toxic effects of irinotecan on hepatocytes. 
Table I. RT-PCR primer sequences.

\begin{tabular}{|c|c|c|}
\hline Gene & $5^{\prime}$-Primer $\left(5^{\prime}-3^{\prime}\right)$ & 3'-Primer (5'-3') \\
\hline Hmgcr & GCCCAAAATTTGAAGAGGACGTG & CCGAGAAAGCTCTAGGACCAGGG \\
\hline Idil & TGAAAACATTGACAAAGGACTAATACATCGAG & TCATTTCATTTAGATCAACCTCTTCCAAGG \\
\hline Sqle & TCCAAGAGGCGCAGAAAAGAAGTC & TGTATCTCCAAGGCCCAGCTCTC \\
\hline Cyp7al & GAATTGCCGTGTTGGTGAGCTG & GCTTCTGTGTCCAAATGCCTTCG \\
\hline Abcg5 & TTCAGCGTCAGCAACCGTGTC & TGTCAGGACTGCCTCTACCTTCTTGTC \\
\hline$A b c b 11$ & TTTTCCAGAGGCAGCTATCG & ATGGCTGCACTCAAAGATCC \\
\hline Slc10al & AGGCATGATCATCACCTTCC & AAGTGGCCCAATGACTTCAG \\
\hline$U g t 2 b$ & ACATTTTACAGTGAGATTTTGGGAAGGC & AGGATGTCATTCTGCGGGAGC \\
\hline Fasn & TCCCAGGTCTTGCCGTGC & GCGGATGCCTAGGATGTGTGC \\
\hline $\operatorname{Acss} 2$ & GCTTTTTACTGGGAGGGCAATGAG & ССTTCTCTCGGCACTTCTCCAAG \\
\hline $\operatorname{Acsm} 5$ & GCTTGTATGCGAACAGGTGTGGTC & CCTTCCACTGGCCACAAAACC \\
\hline Acsl5 & ATTGAGGGAGGAGCACGGAGAG & TCAGCTCTGTTGATGACATAGATGATGG \\
\hline Elovl6 & CTCTTGCGGTCTTCAGTATATTCGGTG & TCCTCAGAATGATGAATATCGTATCACCTAGTTC \\
\hline Egrl & AGCACCTGACCACAGAGTCCTTTTC & ACGGCACGGCACAGCTC \\
\hline Cxcl9 & TCGAGGAACCCTAGTGATAAGGAATCAG & TTTGCTTTTTCTTTTGGCTGATCTTTTTC \\
\hline $\operatorname{Ly} 6 c$ & GTGTGCAGAAAGAGCTCAGGGC & TGTCCGTCTTACAGAGCCCTCTACAG \\
\hline$\beta$-actin & GTACCACTGGCATTGTGATG & ATCTTCATGGTGCTAGGAGC \\
\hline
\end{tabular}

Fasn, fatty acid synthase.

Nonalcoholic steatohepatitis (NASH) is also characterized by similar pathological findings to those observed in irinotecan-associated steatohepatitis (13). This lesion shows lipid accumulation in parenchymal cells and alterations in lipid metabolism are suggested as factors involved in its pathogenesis $(14,15)$. Kupffer cells have been shown to promote the inflammatory response associated with NASH by releasing proinflammatory mediators (16).

To explore the mechanism underlying irinotecan-associated hepatic lesions, we investigated factors contributing to their pathogenesis using an animal model. The results obtained in the present study revealed that hepatic steatosis occurred on day 18 after the last injection of irinotecan and was preceded by fatty acid synthetase strongly-positive hepatocytes and the activation of liver progenitor cells.

\section{Materials and methods}

Animals and animal treatment. Male Sprague-Dawley rats weighing 170-230 g, aged 6 weeks, were purchased from Clea Japan, Inc. (Shizuoka, Japan). These rats were kept under routine laboratory conditions at the animal laboratory of Hirosaki University. The rats received standard laboratory chow, had free access to food and water and were kept in a thermostatically controlled room $\left(25^{\circ} \mathrm{C}\right)$ with a 12 -h lightdark cycle. Rats were administered $200 \mathrm{mg} / \mathrm{kg}$ irinotecan by intraperitoneal injection on days 1-4, but not treated on days 5,6 or 7 . This schedule was repeated 3 times. Rats were sacrificed 4, 18 and 25 days after the last injection and livers were removed for analysis. Control rat received $2 \mathrm{ml} /$ body saline intraperitoneal injections under the same schedule, and were sacrificed on day 18 . Three rats were used for each time point. The present study was carried out in accor- dance with the Guidelines for Animal Experimentation of Hirosaki University, and all animals received humane care according to the criteria outlined in the 'Guide for the Care and Use of Laboratory Animals' prepared by the National Academy of Sciences and published by the National Institutes of Health (NIH publication 86-23, revised 1985).

Microarray analysis. Total RNA was extracted from frozen liver samples with TRIzol reagent (Invitrogen, Carlsbad, CA, USA). Equal amounts of RNA from 3 individual livers were combined and $10 \mu \mathrm{g}$ of RNA was used for biotin-labeled complementary RNA (cRNA). Labeled and fragmented cRNA was subsequently hybridized to the GeneChip Rat Gene 1.0 ST Array (Affymetrix, Santa Clara, CA, USA). Labeling, hybridization, image scanning and data analysis were performed at Kurabo Industries Ltd. (Osaka, Japan).

Quantitative real-time polymerase chain reaction (RT-PCR). Complementary DNA (cDNA) was reverse-transcribed from $1 \mu \mathrm{g}$ of total RNA using the Omniscript RT kit (Qiagen, Tokyo, Japan). A MiniOpticon Detection System and SYBR-Green Supermix (both from Bio-Rad Laboratories, Hercules, CA, USA) were used for the quantification of specific messenger RNA (mRNA). The amplification of $\beta$-actin cDNA was performed to standardize target cDNA levels. Gene-specific primers were designed according to known rat sequences (Table I). No non-specific PCR products, as detected by melting temperature curves, were found in any case. After normalizing the expression of the target gene to the expression of $\beta$-actin, the level of the expressed mRNA in each sample was expressed relative to the control values. 
Immunohistochemistry. Tissue samples of livers were fixed in $10 \%$ neutral buffered formaldehyde and embedded in paraffin. These paraffin blocks were sliced into $4-\mu \mathrm{m}$ thick sections for hematoxylin and eosin (H\&E) staining, periodic acid-Schiff (PAS) staining and immunostaining for carbonic anhydrase 3 (CA3), CD68, CD163, chemokine (C-X-C motif) ligand 9 ( $\mathrm{Cxcl} 9)$, cytokeratin 19 and fatty acid synthase (Fasn). Immunohistochemical staining was performed on deparaffinized sections using the standard avidin-biotin-peroxidase complex method with an automated immunostainer (Benchmark XT; Ventana Medical System, Tucson, AZ, USA). The primary antibodies used were: antiCD68 antibody from AbD serotec (MCA341R) (Oxford, UK), anti-CD163 antibody from Santa Cruz Biotechnology (sc-58965) (Santa Cruz, CA, USA), anti-Cxcl9 antibody from Bioss (bs-2551R) (Boston, MA, USA), anti-cytokeratin 19 antibody from Bioworld Technology, Inc. (BS3540) (St. Louis Park, MN, USA), anti-Fasn antibody from Abcam (ab22759) (Cambridge, UK). Anti-CA3 antibodies were prepared as described by Takahata et al (17). Non-immune $\gamma$-globulin was used for the negative controls instead of the primary antibodies. Images were captured with an Olympus FSX100 microscope (Olympus, Tokyo, Japan). Digital images were processed with Adobe Photoshop (Adobe, San Jose, CA, USA) and ImageJ software (Wayne Rasband NIH, Bethesda, MD, USA). The percentage of intracellular vacuoles and number of Fasn-strongly-positive cells were compared between areas around the central vein $(\mathrm{CV})$ and bile ducts (BD).

Statistical analysis. Data are presented as means \pm SD. Statistical evaluations were performed using the two-tailed Student's t-test. Differences were considered to indicate a significant result with $\mathrm{p}$-values $<0.05$.

\section{Results}

Induction of intrahepatocyte vacuoles after irinotecan injection. Liver sections from rats administered irinotecan were evaluated by H\&E staining and the results are shown in Fig. 1a-d. Although no significant difference was observed between control and day 4 (Fig. 1a and b), panacinar intrahepatocyte vacuoles were detected on day 18 (Fig. 1c), and they had disappeared by day 25 (Fig. 1d). To evaluate the degree of intracellular vacuoles quantitatively, two methods were used. Firstly, we used nonalcoholic fatty liver disease (NAFLD) activity score on H\&E-stained liver sections, according to Kleiner et al (14). The value around CV was $4.7 \pm 0.6$, while that around BD was $6.7 \pm 0.6$ on day 18 . These values were 0 in control and days 4 and 25 in the irinotecan group (Fig. 1e). Another method used was the percentage of intracellular vacuoles per unit area calculated by subtracting sinusoid areas from the unstained areas of H\&E-stained sections. Comparing with the control, this value around BD was significantly increased to $15.6 \pm 2.5$ on day 18 and $7.0 \pm 0.6$ on day 25 (closed bars in Fig. 1f). The value around $\mathrm{CV}$ was lower on day 4 , but was higher at $8.8 \pm 0.4$ on day 18 (open bars). These results suggested that hepatic steatosis was induced by irinotecan administration, prominent on day 18 , and was more severe around $\mathrm{BD}$ than $\mathrm{CV}$.
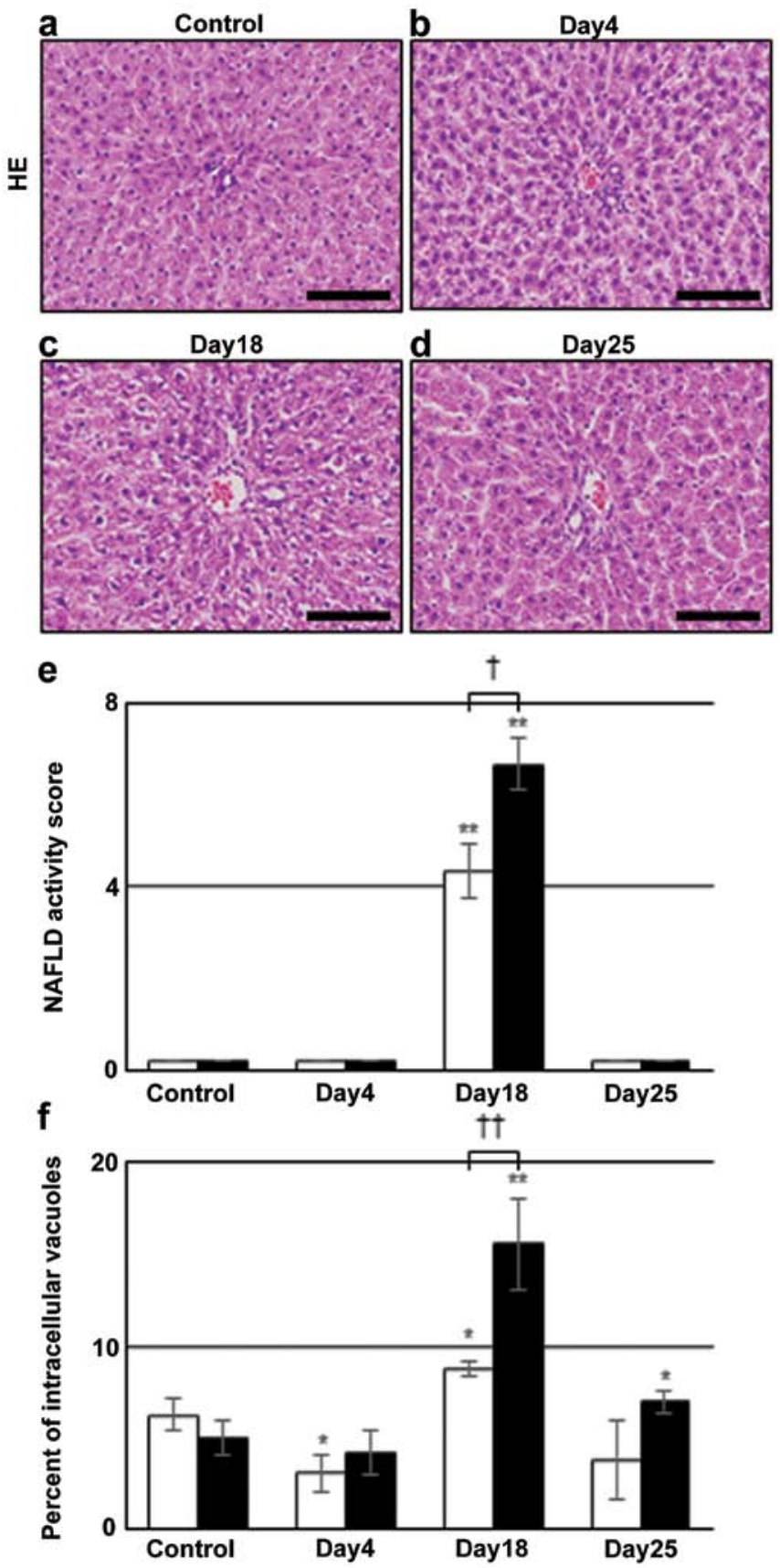

Figure 1. H\&E staining of liver sections and the quantitative evaluation of intracellular vacuoles. (a-d) H\&E staining in (a) control and irinotecan group on days (b) 4, (c) 18 and (d) 25 . The data shown are from a representative preparation set and are similar to results obtained in two other sets. Intracellular vacuoles are markedly increased at day 18. Scale bars represent $100 \mu \mathrm{m}$. (e) Nonalcoholic fatty liver disease (NAFLD) activity score on H\&E-stained liver sections, depending on steatosis grade (0-3), location (0-3) and microvesicular steatosis (0-1). Open bars indicate scores around CV and closed bars those around BD. Data are the mean \pm SD from 3 rats. (f) Percentage of intracellular vacuoles/unit area $\left(0.036 \mathrm{~mm}^{2}\right)$, calculated by subtracting sinusoid areas from the unstained areas of H\&E-stained sections. Data are the mean \pm SD from 3 rats. ${ }^{*} \mathrm{p}<0.05$ vs. control; ${ }^{* *} \mathrm{p}<0.01 \mathrm{vs}$. control (two-tailed Student's t-test). ${ }^{+} \mathrm{p}<0.05$ and ${ }^{+\dagger} \mathrm{p}<0.01$ between values around $\mathrm{CV}$ and those around BD (two-tailed Student's t-test). H\&E, hematoxylin and eosin; CV, central vein; BD, bile ducts.

Intracellular vacuole formation in CA3-positive hepatocytes. The expression of CA3, a marker for lipogenesis (18), was immunohistochemically evaluated to assess the presence of vacuoles in lipid-storing hepatocytes. Many hepatocytes 

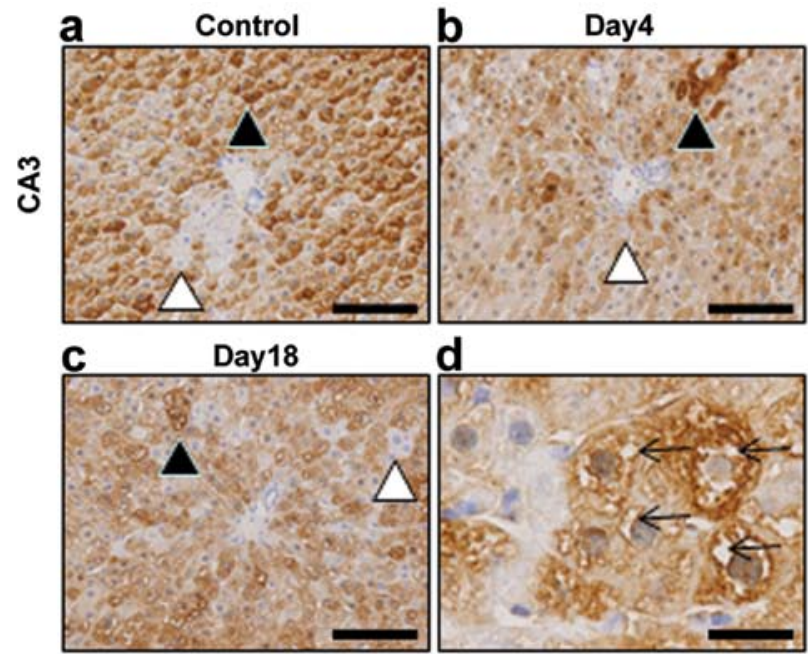

d

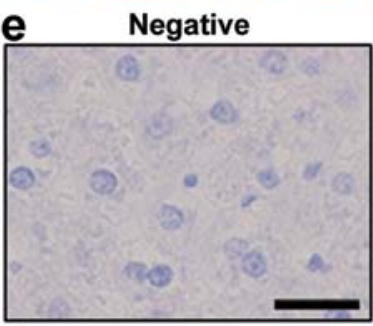

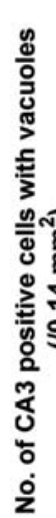

f

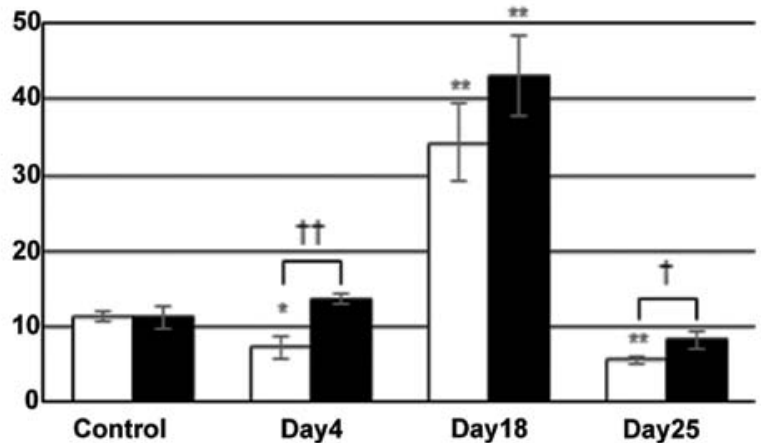

Figure 2. Immunostaining for CA3 in (a) control and irinotecan group on days (b) 4 and (c and d) 18. Liver sections of control group were stained with non-immune (e) $\gamma$-globulin. (d) The panel is at a higher magnification in the (c) panel. Closed and open arrowheads indicate positive and negative hepatocytes, respectively. (d) Arrows in the panel show intracellular vacuoles. The data shown are from a representative preparation set and are similar to results obtained in two other sets. (a-c) Scale bars $100 \mu \mathrm{m}$ and (d and e) $25 \mu \mathrm{m}$. (f) Number of CA3-positive hepatocytes accompanying intracellular vacuoles. Open bars indicate the number around the $\mathrm{CV}$ and closed bars around the BD. Data are the mean \pm SD from 3 rats. ${ }^{*} p<0.05$ vs. control; ${ }^{* *} \mathrm{p}<0.01$ vs. control (two-tailed Student's t-test). ${ }^{\dagger} \mathrm{p}<0.05$ and ${ }^{\dagger+} \mathrm{p}<0.01$ between the values for $\mathrm{CV}$ and for the BD (two-tailed Student's t-test). CA3, carbonic anhydrase $3 ; \mathrm{CV}$, central vein; $\mathrm{BD}$, bile ducts.

were positive for CA3 (closed arrowheads in Fig. 2), but some cells were negative (open arrowheads). The number of CA3-positive hepatocytes was not changed after irinotecan injection. Vacuole formation in hepatocytes was easily detectable by this staining. Such vacuoles in CA3-positive cells were not observed in the control (Fig. 2a), or irinotecan group on day 4 (Fig. 2b) or 25 (data not shown), but were observed on day 18 (Fig. 2c and d). CA3-positive hepatocytes with vacuoles were mainly distributed around $\mathrm{BD}$, which was consistent with the results from H\&E-stained liver sections (Fig. 1).
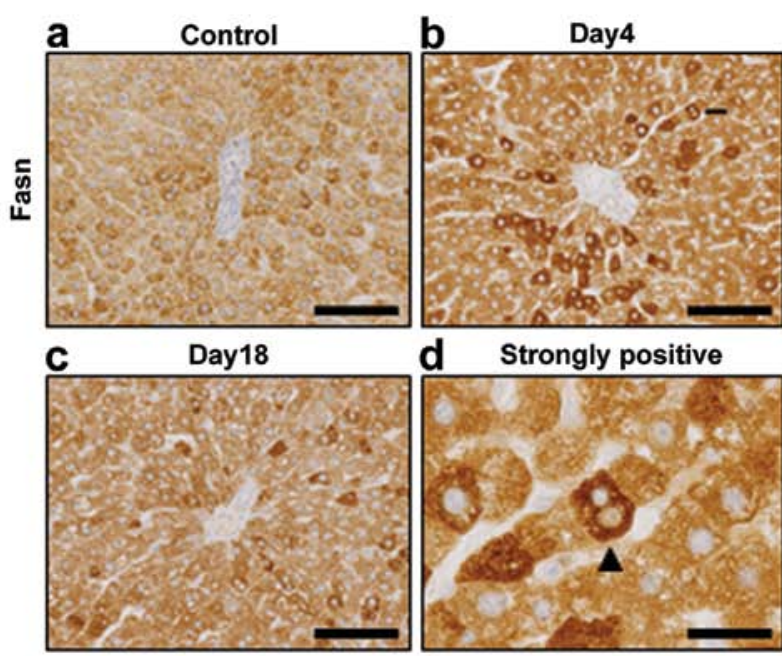

d Strongly positive
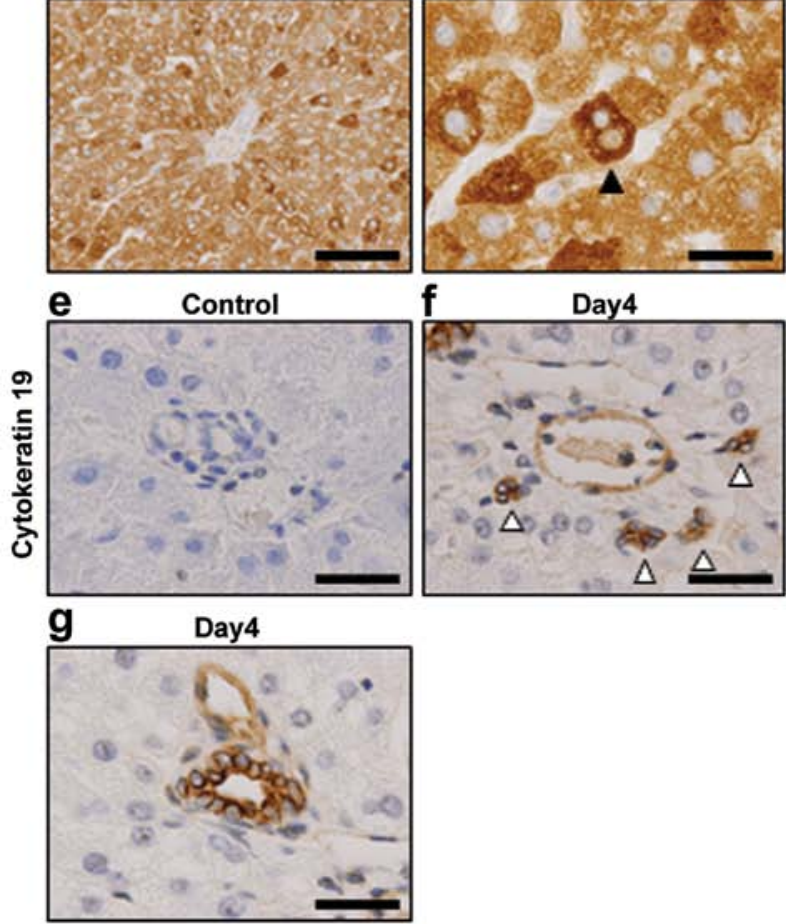

h

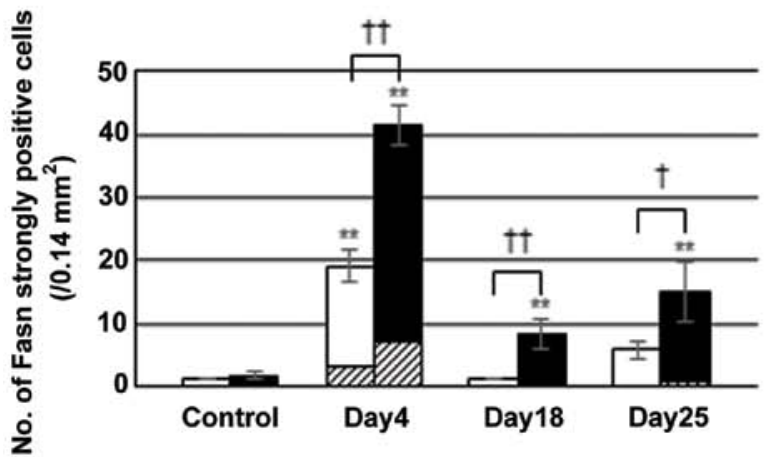

Figure 3. (a-d) Immunostaining for Fasn, and (e-g) cytokeratin 19 in (a and e) control and irinotecan group ( $b, \mathrm{~d}, \mathrm{f}$ and $\mathrm{g}$ ) on day 4 , and the number of (h) Fasn-strongly-positive hepatocytes. (b) An arrow and (d) a closed arrowhead indicate cells accompanying two nuclei, and (f) open arrowheads show cytokeratin 19-positive cells forming clusters. The data shown are from a representative preparation set and are similar to results obtained in two other sets. (a-c) Scale bars $100 \mu \mathrm{m}$, and (d-g) $25 \mu \mathrm{m}$. (h) The number of Fasnstrongly-positive hepatocytes. Open bars indicate the number around the $\mathrm{CV}$ and closed bars around the BD. Hatched bars represent the number of cells with two nuclei. Data are the mean $\pm \mathrm{SD}$ from 3 rats. These cells were quantified from five microscope fields $\left(0.14 \mathrm{~mm}^{2} /\right.$ field $)$ for each rat. ${ }^{* *} \mathrm{p}<0.01 \mathrm{vs}$. control (two-tailed Student's t-test). ${ }^{\dagger} \mathrm{p}<0.05$ and ${ }^{+\dagger} \mathrm{p}<0.01$ between the values for $\mathrm{CV}$ and the BD (two-tailed Student's t-test). Fasn, fatty acid synthase; CV, central vein; $\mathrm{BD}$, bile ducts.

Enhanced expression of genes for cholesterol and fatty acid synthesis. Gene expression profiles were examined using 
Table II. The results of microarray analysis in control and irinotecan group at days 4, 18 and 25 .

Signal

Cellular function and gene name

Gene

\begin{tabular}{llll}
\multicolumn{4}{c}{ Signal } \\
\hline Control & Day 4 & Day 18 & Day 25
\end{tabular}

Cholesterol synthesis

Citrate

ATP-citrate lyase

symbol

Control

Day 4

Day 25

Acetyl-CoA

Acetoacetyl-CoA synthetase

HMG-CoA synthase 1

HMG-CoA reductase

$\begin{array}{lrrrr}\text { Acly } & 2,096 & 5,121 & 2,511 & 1,700 \\ & & & & \\ \text { Aacs } & 91 & 1,462 & 309 & 226 \\ \text { Hmgcs1 } & 2,691 & 8,433 & 4,088 & 5,586 \\ \text { Himgcr } & 1,001 & 5,012 & 1,878 & 1,276 \\ & & & & \\ \text { Mvd } & 265 & 1578 & 495 & 478 \\ \text { Idil } & 488 & 2467 & 675 & 933 \\ & & & & \\ \text { Sqle } & 362 & 2,980 & 980 & 870 \\ & & & & \\ \text { Cyp51 } & 3,430 & 8,418 & 4,975 & 4,194 \\ \text { Dhcr7 } & 1,408 & 4,582 & 1,912 & 1,996 \\ & & & & \\ \text { Cyp7al } & 1,242 & 7,299 & 1,671 & 1,250 \\ \text { Cyp27al } & 2,228 & 5,076 & 2,781 & 3,078\end{array}$

Mevalonate

Mevalonate pyrophosphate decarboxylase

Isopentenyl-diphosphate $\Delta$-isomerase 1

Squalene

Squalene epoxidase

Cyp27a1

2,228

3,078

Cholesterol and bile acid export

Bile canaliculus

ATP-binding cassette sub-family $\mathrm{G}$ member 5

Abcg 5

Abcg8

515

1,345

827

542

ATP-binding cassette sub-family $\mathrm{G}$ member 8

222

794

476

169

Sinusoid

ATP-binding cassette, sub-family C (CFTR/MRP), member 3

Abcc3

230

1,583

595

297

Bile acid conjugation

UDP glucuronosyltransferase 2 family, polypeptide A3

Ugt2a3

1,852

3,972

2,538

2,912

UDP glucuronosyltransferase 2 family, polypeptide B

Ugt $2 b$

6,163

4,034

4,731

Fatty acid synthesis

Fatty acid synthase

Elongation of long chain fatty acid member 6

Fasn

2,096

5,140

2,650

1,687

Elovl6

461

5,188

500

571

Acyl-CoA synthetase

Acyl-CoA synthetase short-chain family member 2

Acyl-CoA synthetase medium-chain family member 2

Acyl-CoA synthetase long-chain family member 5

$\operatorname{Acss} 2$

1,367

6,140

2,190

1,892

Acsm2

251

1,199

820

213

Acsl5

3,079

5,723

4,308

3,181

Transcription factor

Sterol regulatory element-binding factor 2

Srebf2

1,068

1,826

1,365

1,358

Insig1

1,734

6,462

3,397

3,819

SREBP cleavage-activating protein

Scap

888

1,779

1,014

900

Early growth response 1

Nuclear receptor subfamily 1, group I, member 3

Egrl

629

2,932

1,308

Nrli3

DNA

Cell cycle

Cyclin B1

Cyclin-dependent kinase inhibitor 3 
Table II. Continued.

Signal

Cellular function and gene name

Gene

DNA replication

Ribonucleotide reductase M2

symbol

Control Day 4 Day 18

Day 25

Topoisomerase (DNA) II $\alpha$

$\begin{array}{lrrrr}\text { Rrm2 } & 156 & 60 & 378 & 534 \\ \text { Top2a } & 154 & 71 & 391 & 376 \\ & & & & \\ \text { Cdc20 } & 165 & 103 & 293 & 343 \\ \text { Ckap2 } & 77 & 33 & 175 & 154 \\ & & & & \\ \text { Eif2ak4 } & 314 & 549 & 402 & 337 \\ & & & & \\ \text { Cd68 } & 612 & 722 & 654 & 761 \\ \text { Cd163 } & 747 & 831 & 713 & 691 \\ \text { Mrc1 } & 1,383 & 1,408 & 1,317 & 1,402 \\ \text { Cxcl1 } & 251 & 710 & 540 & 371 \\ \text { Cxcl9 } & 1,118 & 282 & 423 & 923\end{array}$

Mitosis

Cell division cycle 20 homolog (S. cerevisiae)

Cytoskeleton associated protein 2

ER stress

Eukaryotic translation initiation factor $2 \alpha$ kinase 4

Kupffer cells

CD68 molecule

CD163 molecule

Mannose receptor, $\mathrm{C}$ type 1

Chemokine (C-X-C motif) ligand 1

Chemokine (C-X-C motif) ligand 9

Cxcl9

1,118

Ly6c

219

144

500

560

Sinusoids

CD34 molecule

$C d 34$

100

93

98

101

Stellate cells

Collagen, type I, $\alpha 1$

Collal

320

395

582

397

Collagen, type I, $\alpha 2$

Colla2

426

532

610

405

Desmin

Des

171

116

108

Liver progenitor cells

Cytokeratin 19

Krt19

111

224

155

115

Epithelial cell adhesion molecule

Epcam

236

545

242

185

microarray analysis in order to identify the genes responsible for inducing steatosis (Table II) (19). This revealed that the expression of genes involved in the synthesis of cholesterol and fatty acids was upregulated on day 4. The upregulated genes for the synthesis of cholesterol include Acly, Hmgcr, Mvd, Sqle and Dhcr7. Genes for the synthesis (Cyp7a1), export (Abcg5 and $A b c c 3$ ), and conjugation (Ugt2a3 and $U g t 2 b)$ of bile acids were also upregulated. The expression of genes for the metabolism of fatty acids [Fasn (20), Elovl6 and Acss2) was also enhanced. Sterol regulatory element-binding proteins 1 and 2 (Srebp1 and 2) have been shown to regulate the transcription of genes involved in the synthesis of cholesterol and fatty acids (21), while insulin-induced gene 1 (Insig1) and SREBP cleavage-activating protein (Scap) mediate the activation of Srebp1 and 2 (22,23). Insigl and Scap were upregulated on day 4 . The expression of other transcription factors involved in lipid metabolism, Egrl (24) and Nrli3 (CAR) (25), was unchanged on day 4 , yet upregulated by day 18 . The expression of genes associated with the cell cycle and DNA replication (Ccnb1, Rrm2 and Top2a) was downregulated on day 4, yet upregulated on days 18 and 25. A gene related to endoplasmic reticulum stress, eukaryotic translation initiation factor $2 \alpha$ kinase 4 (Eif2ak4) (26) was upregulated on days 4 and 18. The expression of Kupffer cell marker genes, except for $\mathrm{Cxcl}$, remained unchanged. No significant differences were observed in the expression of sinusoid and stellate cell marker genes. The expression of the liver progenitor cell marker genes, Krt19 and Epcam, was upregulated on day 4. To confirm the microarray results, some mRNA levels of cholesterol, bile acid and fatty acid synthesis were quantified by RT-PCR (Table III). Hmgcr, Sqle and Cyp7al mRNA were increased on day 4, as compared with control values. Fasn and Acss 2 mRNA levels were increased on days 4 and 18. Egrl mRNA was markedly increased on day 18. Cxcl9 was downregulated on days 4 and 18 .

Appearance of Fasn-strongly-positive hepatocytes. Immunostaining was performed to examine whether Fasn protein levels also increased. Most hepatocytes in the controls were weakly positive for Fasn (Fig. 3a), whereas strongly positive hepatocytes appeared around the BD on day 4 in the irinotecan group (Fig. 3b) and frequently included cells with two nuclei (Fig. 3b). Fasn-strongly-positive cells were decreased on day 18 (Fig. 3c). Polykaryonic cells were also observed in 
Table III. The mRNA by RT-PCR evaluation for cholesterol and fatty acid metabolism in control and irinotecan group.

\begin{tabular}{|c|c|c|c|c|c|}
\hline \multirow[b]{2}{*}{ Cellular function and gene name } & \multirow{2}{*}{$\begin{array}{l}\text { Gene } \\
\text { symbol }\end{array}$} & \multicolumn{4}{|c|}{ Signal } \\
\hline & & Control & Day 4 & Day 18 & Day 25 \\
\hline \multicolumn{6}{|l|}{ Cholesterol synthesis } \\
\hline HMG-CoA reductase & Hmgcr & 1 & 10.14 & 1.07 & 1.97 \\
\hline Isopentenyl-diphosphate $\Delta$-isomerase 1 & Idil & 1 & 9.9 & 1.07 & 0.92 \\
\hline Squalene epoxidase & Sqle & 1 & 55.19 & 12.54 & 6.7 \\
\hline \multicolumn{6}{|l|}{ Bile acid synthesis } \\
\hline Cytochrome P450, family 7, subfamily a, polypeptide 1 & Cyp7al & 1 & 7.73 & 1.91 & 0.59 \\
\hline \multicolumn{6}{|l|}{ Cholesterol and bile acid export } \\
\hline ATP-binding cassette, sub-family $\mathrm{G}$ member 5 & Abcg5 5 & 1 & 1.17 & 1.88 & 0.55 \\
\hline ATP-binding cassette, sub-family B (MDR/TAP), member 11 & $A b c b 11$ & 1 & 1.07 & 1.02 & 1.11 \\
\hline $\begin{array}{l}\text { Solute carrier family } 10 \text { (sodium/bile acid co-transporter family), } \\
\text { member } 1\end{array}$ & SlclOal & 1 & 1.24 & 0.87 & 1.84 \\
\hline \multicolumn{6}{|l|}{ Bile acid conjugation } \\
\hline UDP glucuronosyltransferase 2 family, polypeptide B & $U g t 2 b$ & 1 & 7,362 & 3,310 & 2,392 \\
\hline \multicolumn{6}{|l|}{ Fatty acid synthesis } \\
\hline Fatty acid synthase & Fasn & 1 & 3.58 & 3.85 & 0.81 \\
\hline Acyl-CoA synthetase short-chain family member 2 & $\operatorname{Acss} 2$ & 1 & 12.11 & 6.9 & 2.14 \\
\hline Acyl-CoA synthetase medium-chain family member 5 & $\operatorname{Acsm} 5$ & 1 & 11.94 & 22.49 & 6.03 \\
\hline Acyl-CoA synthetase long-chain family member 5 & Acsl5 & 1 & 5.53 & 6.74 & 1.99 \\
\hline Elongation of long chain fatty acid member 6 & Elovl6 & 1 & 20.23 & 1.19 & 0.55 \\
\hline \multicolumn{6}{|l|}{ Transcription factor } \\
\hline Early growth response 1 & Egrl & 1 & 3.55 & 23.32 & 6.16 \\
\hline \multicolumn{6}{|l|}{ Kupffer cells } \\
\hline Chemokine (C-X-C motif) ligand 9 & Cxcl9 & 1 & 0.2 & 0.34 & 0.51 \\
\hline \multicolumn{6}{|l|}{ Monocytes } \\
\hline Ly6-C antigen & $\operatorname{Ly} 6 c$ & 1 & 0.55 & 1.26 & 1.62 \\
\hline
\end{tabular}

mRNA levels in the irinotecan group are expressed relative to the values of individual mRNAs in the control group.

Fasn-weakly-positive hepatocytes on day 4, but at a lower frequency. The size of polykaryonic cells heavily stained for Fasn was smaller than that of weakly stained cells (Fig. 3d).

Activation of liver progenitor cells. Immunohistochemistry was performed for cytokeratin 19 in order to examine alterations in liver progenitor cells. Althougha positive reaction was not obtained in the control (Fig. 3e), liver progenitor cells that formed clusters around portal veins (Fig. 3f) were detected on day 4 in the irinotecan group. In addition, bile duct epithelial cells also showed positive reaction (Fig. 3g). These results demonstrated the activation of liver progenitor cells following the administration of irinotecan, whereas their morphology and localization differed from those of Fasn-strongly-positive hepatocytes (Fig. 3b).

Relationship between Fasn-strongly-positive hepatocytes and CA3-positive hepatocytes with vacuoles. To examine the relationship between Fasn-strongly-positive cells and CA3-positive hepatocytes accompanying intracellular vacu- oles, the localization of these cells was compared. Both cell types were frequently distributed around the BD, but their locations differed (Figs. $3 b$ and 2c). The numbers of Fasnstrongly-positive hepatocytes around the BD increased to $41.3 \pm 3.1$ on day 4 , and then decreased to $8.3 \pm 2.5$ on day 18 (Fig. 3h). Approximately 17\% of Fasn-strongly-positive hepatocytes possessed two nuclei (Fig. 3h) on day 4. The number of CA3-positive hepatocytes accompanying intracellular vacuoles around BD was $13.7 \pm 0.6$ on day 4 , and this increased to $43.0 \pm 5.3$ on day 18 (Fig. $2 \mathrm{f}$ ). Thus, the magnitude of the decrease in the number of Fasn-strongly-positive hepatocytes between days 4 and 18 was similar to that of the increase in the number of CA3-positive hepatocytes accompanying intracellular vacuoles. Similar results were also observed in the values around the $\mathrm{CV}$ between days 4 and 18 (Figs. 3h and 2f).

Loss of Kupfer cell marker proteins. To examine the effects of irinotecan on Kupffer cells, the expression of their marker proteins, Cxc19 and CD163, was evaluated by immunohis- 

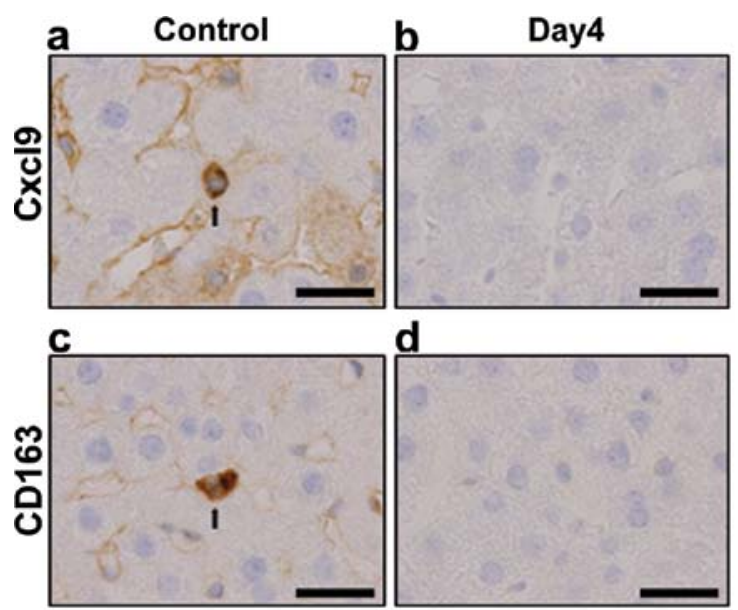

d

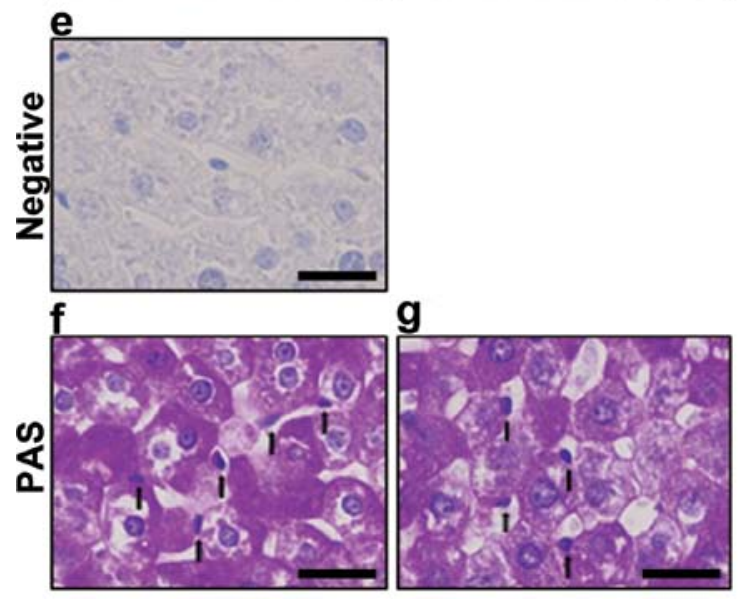

h
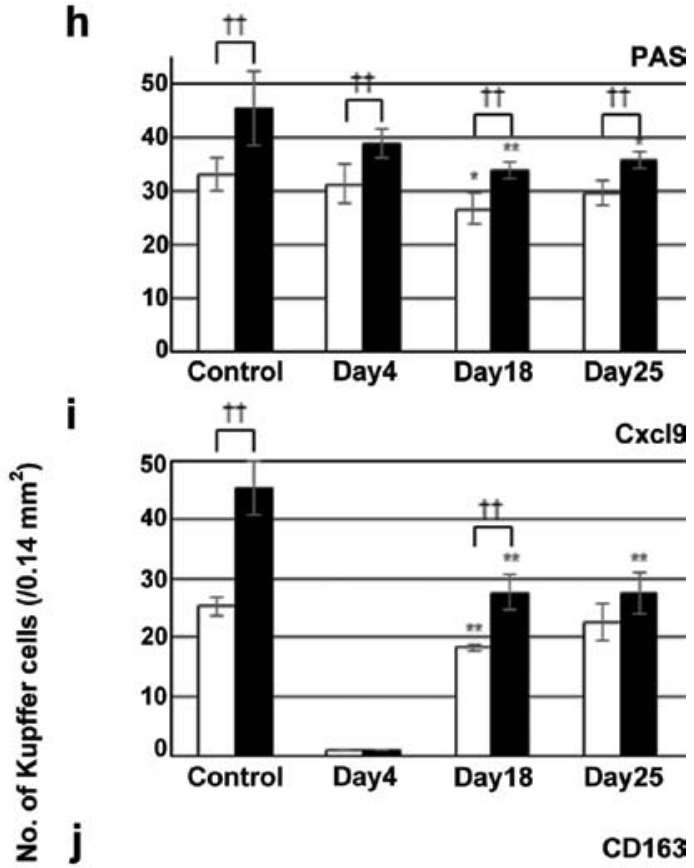

CD163

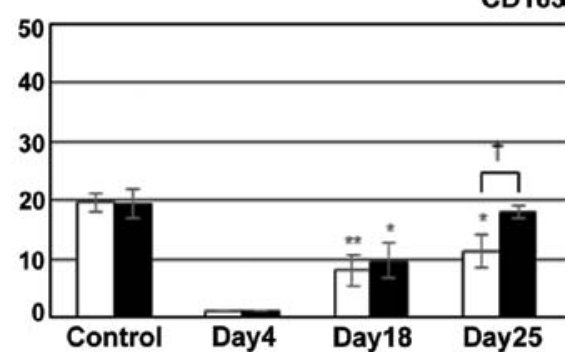

Figure 4. Immunostaining for (a and b) Cxc19, (c and d) CD163 and (f and g) PAS staining in (a, c, e and f) control and irinotecan group on (b, d and g) day 4. Liver sections of control group were also stained with (e) non-immune $\gamma$-globulin. (a, c, f and g) Arrows indicate positive-Kupffer cells. The data shown are from a representative preparation set and are similar to results obtained in two other sets. Scale bars represent $25 \mu \mathrm{m}$. The number of Kupffer cells counted with (h) PAS staining and immunostaining for (i) Cxc19 and (j) CD163 in control and irinotecan group on days 4, 18 and 25. Open bars indicate values around the $\mathrm{CV}$ and closed bars around the $\mathrm{BD}$. Data are the mean $\pm \mathrm{SD}$ from 3 rats. These cells in liver sections were from five microscope fields $\left(0.14 \mathrm{~mm}^{2} /\right.$ field $)$ for each rat. ${ }^{*} \mathrm{p}<0.05$ vs. control; ${ }^{* *} \mathrm{p}<0.01$ vs. control (two-tailed Student's t-test). ${ }^{\dagger} \mathrm{p}<0.05$ between values around the $\mathrm{CV}$ and those around the BD; ${ }^{\dagger} \mathrm{p}<0.01$ between values for the CV and for the BD (two-tailed Student's t-test). Cxcl9, chemokine (C-X-C motif) ligand 9; PAS, periodic acid-Schiff; CV, central vein; $\mathrm{BD}$, bile ducts.

tochemistry. As shown in Fig. 4a, Kupfer and sinusoidal endothelial cells were positive for the anti-Cxc19 antibody in the control, but were negative on day 4 (Fig. 4b), and became positive again on days 18 and 25 (data not shown). Immunostaining for CD163 and CD68 was also negative on day 4 (Fig. 4c and d for CD163, and data not shown for CD68). Non-immune $\gamma$-globulin, instead of the primary antibodies, was also negative (Fig. 4e). On the other hand, PAS staining revealed the presence of Kupffer cells on day 4 (Fig. 4g) as well as in the control (Fig. 4f). Therefore, the results of immunostaining indicated the loss of Kupffer cell markers, but the cells were still present on day 4 . The numbers of Kupffer cells detected by PAS staining were higher around the BD than the CV at each time point (Fig. 4h). The number of Cxcl9positive Kupffer cells around the $\mathrm{BD}$ was also higher than that around the CV in the control (Fig. 4i), whereas that of CD163positive cells was similar between the two areas (Fig. 4j). Cxc19-positive Kupffer cells decreased to 0 on day 4, and then increased to $27.7 \pm 3.1$ around $\mathrm{BD}$ on day 18 , but was still lower than the value obtained in the control (Fig. 4i). CD163-positive Kupfer cells also decreased to 0 on day 4 , and then increased to $9.7 \pm 3.1$ around BD on day 18 (Fig. $4 \mathrm{j}$ ).

\section{Discussion}

Although H\&E staining of liver sections and immunohistochemistry for CA3 revealed no significant changes on day 4, panacinar intrahepatocyte vacuoles were present on day 18 , and had disappeared by day 25 . Microarray and RT-PCR analyses showed that the expression of genes involved in the synthesis of cholesterol and fatty acids was upregulated on day 4 . Thus, genes involved in the synthesis of fatty acids were upregulated on day 4 , and hepatic steatosis appeared on day 18. Immunostaining detected the appearance of Fasn-strongly-positive hepatocytes on day 4 . Some cells possessed two nuclei and were small in size. Since the expression of Fasn is reported in proliferating cells (27), the results of the present study suggested that Fasn-strongly-positive hepatocytes were mitotic. The magnitude of the decrease in the number of Fasn-strongly-positive hepatocytes between days 4 and 18 was similar to that of the increase in the number of CA3-positive hepatocytes accompanying intracellular vacuoles, suggesting that the former cells changed into the latter cells. The degree of steatosis was more prominent around the BV than the $\mathrm{CV}$ and this may have been related to the preferential localization of Fasn-strongly-positive hepatocytes. 
Irinotecan has been identified as an inhibitor of topoisomerase 1, an enzyme involved in DNA replication (10); therefore, withdrawal of the drug may have induced cell proliferation. However, microarray analysis revealed that the expression of genes involved in cell proliferation was downregulated on day 4. Moreover, liver progenitor cells, known as oval cells in rodents, were previously shown to proliferate around the portal vein in response to suppression of hepatocyte proliferation by some agents, to form ductular structures and then expanded into liver parenchyma $(28,29)$. Activation of progenitor cells was demonstrated by immunohistochemistry for cytokeratin 19 in the present study. A previous study reported that the activation of liver progenitor cells was correlated with progression toward NASH (30). This raises a possibility that activated progenitor cells may differentiate into Fasn-strongly-positive hepatocytes. However, both cells were found to be morphologically different and cells that shared the properties of both cell types were not detected. Thus, it is unlikely that such progenitor cells were directly changed into Fasn-strongly-positive cells.

Kupffer cells were previously shown to promote steatohepatitis by enhancing hepatic lipid accumulation through various inflammatory mediators, including specific cytokines (31). However, Kupffer cell dysfunction is also known to induce nonalcoholic fatty liver disease (32). Immunostaining for Cxc19 and CD163 indicated that Kupffer cell marker proteins were absent on day 4, even though Kupffer cells were present. Such protein loss appeared to be specific to Kupffer cells, since CA3 and Fasn proteins were retained in hepatocytes. The expression of Eif $2 a k 4$ gene, which mediates stress responses that suppress global protein synthesis (33) was upregulated on day 4. Kupffer cell dysfunction may be partly involved in the appearance of Fasn-strongly-positive hepatocytes. The number and localization of Cxc19-positive Kupffer cells differed from those of CD163-positive Kupffer cells. As CD163 is a marker for M2 macrophages (34), CD163-positive cells may belong to M2 Kupffer cells (35), while Cxc19-positive cells seem to belong to the M1 cells.

In summary, hepatic steatosis was induced by irinotecan on day 18 and preceded by Fasn-strongly-positive hepatocytes and the activation of liver progenitor cells. The former cells are suggested to change into lipid-accumulating hepatocytes.

\section{Acknowledgements}

The authors would like to thank Dr Takahito Toba and Ms. Yukie Fujita, Department of Pathology and Bioscience, Hirosaki University Graduate School of Medicine, for their technical assistance in immunostaining, and Daiichi-Sankyo Co., Ltd., Tokyo, Japan, for donating irinotecan. The present study was supported in part by a Grant-in-Aid for the Training of Cancer Professionals from the Ministry of Education, Culture, Sports, Science and Technology of Japan.

\section{References}

1. Ohlsson B and Pålsson B: Follow-up after colorectal cancer surgery. Acta Oncol 42: 816-826, 2003.

2. Khatri VP, Petrelli NJ and Belghiti J: Extending the frontiers of surgical therapy for hepatic colorectal metastases: Is there a limit? J Clin Oncol 23: 8490-8499, 2005.
3. Fong Y, Kemeny N, Paty P, Blumgart LH and Cohen AM: Treatment of colorectal cancer: Hepatic metastasis. Semin Surg Oncol 12: 219-252, 1996.

4. Mathijssen RH, van Alphen RJ, Verweij J, Loos WJ, Nooter K, Stoter G and Sparreboom A: Clinical pharmacokinetics and metabolism of irinotecan (CPT-11). Clin Cancer Res 7: 2182-2194, 2001.

5. Carrato A, Gallego J and Díaz-Rubio E: Oxaliplatin: Results in colorectal carcinoma. Crit Rev Oncol Hematol 44: 29-44, 2002.

6. Douillard JY, Cunningham D, Roth AD, et al: Irinotecan combined with fluorouracil compared with fluorouracil alone as first-line treatment for metastatic colorectal cancer: A multicentre randomised trial. Lancet 355: 1041-1047, 2000.

7. Bismuth H, Adam R, Lévi F, Farabos C, Waechter F, Castaing D, Majno P and Engerran L: Resection of nonresectable liver metastases from colorectal cancer after neoadjuvant chemotherapy. Ann Surg 224: 509-522, 1996.

8. Fernandez FG, Ritter J, Goodwin JW, Linehan DC, Hawkins WG and Strasberg SM: Effect of steatohepatitis associated with irinotecan or oxaliplatin pretreatment on resectability of hepatic colorectal metastases. J Am Coll Surg 200: 845-853, 2005.

9. Vauthey JN, Pawlik TM, Ribero D, et al: Chemotherapy regimen predicts steatohepatitis and an increase in 90-day mortality after surgery for hepatic colorectal metastases. J Clin Oncol 24: 2065-2072, 2006.

10. Houghton PJ, Cheshire PJ, Hallman JC, Bissery MC, Mathieu-Boué A and Houghton JA: Therapeutic efficacy of the topoisomerase I inhibitor 7-ethyl-10-(4-[1-piperidino]1-piperidino)-carbonyloxy-camptothecin against human tumor xenografts: Lack of cross-resistance in vivo in tumors with acquired resistance to the topoisomerase I inhibitor 9-dimethylaminomethyl-10-hydroxycamptothecin. Cancer Res 53: 2823-2829, 1993

11. Innocenti $\mathrm{F}$, Undevia $\mathrm{SD}$, Iyer $\mathrm{L}$, et al: Genetic variants in the UDP-glucuronosyltransferase 1A1 gene predict the risk of severe neutropenia of irinotecan. J Clin Oncol 22: 1382-1388, 2004.

12. Welsh FK, Tilney HS, Tekkis PP, John TG and Rees M: Safe liver resection following chemotherapy for colorectal metastases is a matter of timing. Br J Cancer 96: 1037-1042, 2007.

13. Yeh MM and Brunt EM: Pathology of nonalcoholic fatty liver disease. Am J Clin Pathol 128: 837-847, 2007.

14. Kleiner DE, Brunt EM, Van Natta M, et al: Nonalcoholic Steatohepatitis Clinical Research Network: Design and validation of a histological scoring system for nonalcoholic fatty liver disease. Hepatology 41: 1313-1321, 2005.

15. Dorn C, Riener MO, Kirovski G, Saugspier M, Steib K, Weiss TS, Gäbele E, Kristiansen G, Hartmann A and Hellerbrand C: Expression of fatty acid synthase in nonalcoholic fatty liver disease. Int J Clin Exp Pathol 3: 505-514, 2010.

16. Baffy G: Kupffer cells in non-alcoholic fatty liver disease: The emerging view. J Hepatol 51: 212-223, 2009.

17. Takahata T, Kumano T, Ookawa K, Hayakari M, Kakizaki I and Tsuchida S: Inhibition of 3T3-L1 adipocyte differentiation by 6-ethoxyzolamide: Repressed peroxisome proliferator-activated receptor gamma mRNA and enhanced CCAAT/enhancer binding protein beta mRNA levels. Biochem Pharmacol 67: 1667-1675, 2004.

18. Lynch CJ, Fox H, Hazen SA, Stanley BA, Dodgson S and Lanoue KF: Role of hepatic carbonic anhydrase in de novo lipogenesis. Biochem J 310: 197-202, 1995.

19. Yue H, Eastman PS, Wang BB, et al: An evaluation of the performance of cDNA microarrays for detecting changes in global mRNA expression. Nucleic Acids Res 29: E41-E1, 2001.

20. Wakil SJ: Fatty acid synthase, a proficient multifunctional enzyme. Biochemistry 28: 4523-4530, 1989.

21. Horton JD, Goldstein JL and Brown MS: SREBPs: Activators of the complete program of cholesterol and fatty acid synthesis in the liver. J Clin Invest 109: 1125-1131, 2002.

22. Raghow R, Yellaturu C, Deng X, Park EA and Elam MB: SREBPs: The crossroads of physiological and pathological lipid homeostasis. Trends Endocrinol Metab 19: 65-73, 2008.

23. Sakai J, Rawson RB, Espenshade PJ, Cheng D, Seegmiller AC, Goldstein JL and Brown MS: Molecular identification of the sterolregulated luminal protease that cleaves SREBPs and controls lipid composition of animal cells. Mol Cell 2: 505-514, 1998

24. Gokey NG, Lopez-Anido C, Gillian-Daniel AL and Svaren J: Early growth response 1 (Egrl) regulates cholesterol biosynthetic gene expression. J Biol Chem 286: 29501-29510, 2011.

25. Wada T, Gao J and Xie W: PXR and CAR in energy metabolism. Trends Endocrinol Metab 20: 273-279, 2009. 
26. Baird TD and Wek RC: Eukaryotic initiation factor 2 phosphorylation and translational control in metabolism. Adv Nutr 3: 307-321, 2012.

27. Pandey PR, Liu W, Xing F, Fukuda K and Watabe K: Anti-cancer drugs targeting fatty acid synthase (FAS). Recent Patents Anticancer Drug Discov 7: 185-197, 2012.

28. Paku S, Schnur J, Nagy P and Thorgeirsson SS: Origin and structural evolution of the early proliferating oval cells in rat liver. Am J Pathol 158: 1313-1323, 2001.

29. Yovchev MI, Grozdanov PN, Zhou H, Racherla H, Guha C and Dabeva MD: Identification of adult hepatic progenitor cells capable of repopulating injured rat liver. Hepatology 47: 636-647, 2008.

30. Nobili V, Carpino G, Alisi A, Franchitto A, Alpini G, De Vito R, Onori P, Alvaro D and Gaudio E: Hepatic progenitor cells activation, fibrosis, and adipokines production in pediatric nonalcoholic fatty liver disease. Hepatology 56: 2142-2153, 2012.

31. Odegaard JI, Ricardo-Gonzalez RR, Red Eagle A, Vats D, Morel CR, Goforth MH, Subramanian V, Mukundan L, Ferrante AW and Chawla A: Alternative M2 activation of Kupffer cells by PPARdelta ameliorates obesity-induced insulin resistance. Cell Metab 7: 496-507, 2008.
32. Yang SQ, Lin HZ, Lane MD, Clemens M and Diehl AM: Obesity increases sensitivity to endotoxin liver injury: Implications for the pathogenesis of steatohepatitis. Proc Natl Acad Sci USA 94: 2557-2562, 1997.

33. Dang Do AN, Kimball SR, Cavener DR and Jefferson LS: eIF2alpha kinases GCN2 and PERK modulate transcription and translation of distinct sets of mRNAs in mouse liver. Physiol Genomics 38: 328-341, 2009.

34. Edin S, Wikberg ML, Dahlin AM, Rutegård J, Öberg Å, Oldenborg PA and Palmqvist R: The distribution of macrophages with a M1 or M2 phenotype in relation to prognosis and the molecular characteristics of colorectal cancer. PLoS One 7: e47045, 2012

35. Dixon LJ, Barnes M, Tang H, Pritchard MT and Nagy LE: Kupffer cells in the liver. Compr Physiol 3: 785-797, 2013. 